\title{
Virtual Prototype Technology of Product Which Based on Computer
}

\author{
Xiaoyan Jiang ${ }^{\mathrm{a}}$, Yiming $\mathrm{He}^{\mathrm{b}}$ \\ ${ }^{a}$ School of Electronic Information \& Electric Engineering, Changzhou Institute of Technology, CZU, \\ Changzhou, China \\ ${ }^{b}$ Changzhou Institute of Technology, CZU, Changzhou, China
}

\begin{abstract}
In this paper, we have mainly analyzed the relationship between virtual prototype and concurrent design. Then we have concluded the model of virtual prototype and simulation method which based on field object. On the other hand, we have introduced the key technology which supports virtual prototype's integration framework.
\end{abstract}

Index Terms: Product design; Physical prototype; Digital prototype; Virtual prototype

(C) 2011 Published by MECS Publisher. Selection and/or peer review under responsibility of the Research Association of Modern Education and Computer Science.

\section{Introduction}

The product design \& manufacture department want to improve product design quality efficiently in the circumstance of intense trade competition and complex modern high technology product design. They also want to reduce the periodicity of product design and the cost of design product.

During the process of traditional product design and manufacture, in order to identify the product whole performance, we always use Physical Prototype method. But the periodicity and cost of this kind of method are very high. With the rapid development of computer technology and CIMS technology, Virtual Prototype is playing more and more important role in product design and manufacture process from 1990. Virtual Prototype is a kind of digital model which can be simulated, it is similar to actual product in connection with product design information or function, action which caused by product concept.

\section{Concept of Physical Prototype, Digital Prototype and Virtual Prototype}

Prototype is the original form of product. It doesn't need all characteristics of final product, it only has the needed key characteristics of product, such as shape, physical or functional characteristics. When we design any product, a Prototype Machine is always existed. The so-called Prototype Machine is a kind of physical device which is about some new type or new design. Designers can check the compatibility among all components'

* Corresponding author.

E-mail address: ayanzi fei@163.com 
design characteristics and the design characteristics of whole Prototype Machine. Product Prototype can be divided into physical prototype, digital prototype and virtual prototype.

\subsection{Physical prototype}

In order to compare all kinds of design and manufacture sections, we always need to build a series of product testing models with small proportion or whole proportion. The correctness of product design can be checked by such different experience background people as design, technology, management and market. In order to reflect the characteristics of actual product, this kind of testing model's manufacture needs designer to spend plenty of time and cost. The model even affects the determining of system performance directly. Usually, this kind of model is called physical prototype.[1]

Fig. 1 shows the parallel design of virtual prototype product. When the above structure functions' design and checking have been finished, the virtual prototype of product can be acquired in accordance with product function structure information, library component information and some experience data. Virtual prototype includes the needed system structure and physical design information. We can simulate and test virtual prototype with all kinds of restrictions on the basis of virtual prototype. So we can judge the performance parameters of product whether meet the requirements and the design whether is reasonable by analyzing and planning physical parameters' information. Then the feasibility of product can be known. If the performance parameters can satisfy the requirements, we should propose the corresponding modifying suggestions and rebuild virtual prototype or modify design.

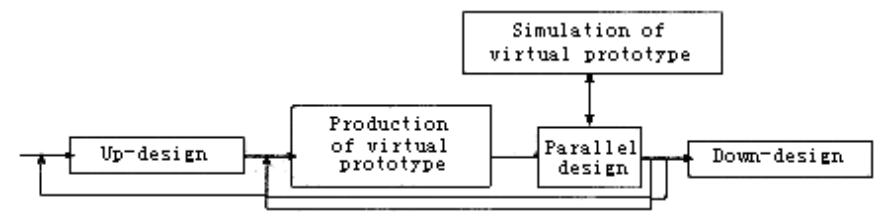

Fig. 1. Product parallel design which used virtual prototype

\subsection{Digital Prototype}

Digital prototype is the product model which designed in accordance with CAD solid modeling software and feature modeling technology. It is a kind of replace technology of physical prototype. On the basis of CAD model, we can take the engineering analysis about finite element, kinematics and dynamics, which checking and improving design effect. All these analyzing programs can provide detailed information about product functions which only used by specialized people. At the original product design stage, we always ignored the detailed analysis. And we paid more attention to design product shape, whole distribution. So, the digital prototype which based on traditional CAD/CAM can meet the requirements.[2]

- Production of virtual prototype

- Parallel design

\subsection{Virtual Prototype}

Virtual prototype finishes the physical prototype machine functions by constructing a digitized prototype machine. It realizes the modeling and analysis functions on the basis of virtual prototype. It combines simulation methods on the basis of CAD model, which provides a kind of method for the building of prototype.

Of course, virtual prototype can't replace the current CAD technology; it works on the basis of CAD data. Virtual prototype can provide additional functional information for the designed object, while product model 
database includes the whole and integrated product model data. This can provide shared information for product development at different stages.[3]

Virtual prototype simulation operates on the function basis of domain object. The model is a network which consists of many domain objects in logical. And they are managed y a server. The user who joins virtual prototype simulation connects server by means of client connection joint. Fig. 2 shows the connection. The core of server connection is object manager. It reflects the products' whole structure information by managing a set of entity objects. The client connection consists of visual object, simulating client agent and coordination virtual prototype and simulation interface. The visual object caused by object governor in accordance with the client's simulation requirements. It recodes the content and form that user wants to get. Its main role is that it can provide the needed product simulation visual picture when it is used to coordinate the simulation of client agent. This can reduce redundant information. User can operate virtual prototype on the basis of interface. After being sensed by virtual prototype interface, the operation can be converted into exciting form which can be recognized by domain object, and then be transmitted to every domain object by means of virtual prototype server.

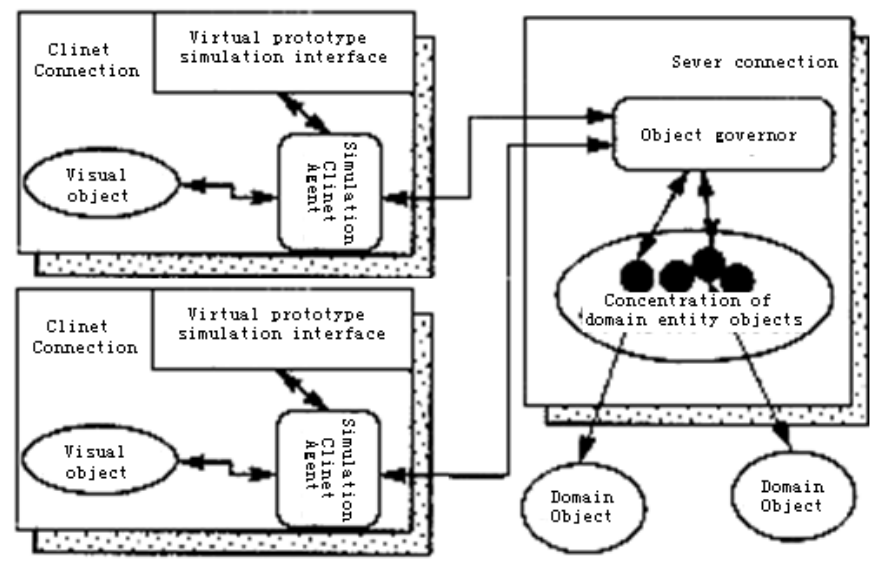

Fig. 2. Model framework which based on domain object

\subsection{Virtual prototype technology}

Virtual prototype technology is a kind of engineering method which can shorten new product development cycles by replacing cost physical prototype with digitized or virtual digital model. United States Department of Defense defined that people acquire physical model design characteristics by building similar model by means of computer simulation technology and testing the model.

The purpose of developing virtual prototype is observing, analyzing and processing product.

\section{Characteristcs of virtual prototype developing method}

Comparing with traditional physical prototype design method, virtual prototype developing method has the following characteristics:

First, it is a new studying mode. Virtual prototype technology has realized product optimization from the angle of system. It can analyze project on the stage of product concept design, compare with many kinds of designs, determine important parameters and forecast the characteristics of product by means of visual technology design. 
Second, its developing cost is lower, its developing circle is shorter and the product quality is better. We can overcome the restriction of cost and time conditions and finish many physical virtual tests by means of computer technology building product digitized model. So, we can acquire the optimal design without making physical machine.

Third, it is the important method which realizing dynamic alliance. Virtual prototype machine is a kind of digitized model. It transmits product information by means of network. It has such characteristics as rapid and timely.

\section{Significiance of virtual product development (VPD)}

The basic thought of virtual product development is that people can finish the whole product developing process by means of computer. Engineer builds product model, analyzes model and improves the design of product by means of computer. VPD replaces traditional material object test with digitized form product by building product's digital model. Then we can analyze product's static and dynamic characteristics on the circumstance of digital state, and rebuild and improve the original design. So, we only need to make one material object prototype.[4]

\section{Main steps about building virtual prototype}

- Getting geometrical model from CAD/CAM model

- Approximating geometrical model by using polyhedron and polygon

- Deleting the unnecessary details in accordance with different requirements

- Editing virtual prototype

- Pasting characteristic outline which express some details better

- Adding circumstance and geometrical model of other factors

- Adding operational functions and characteristics

\section{Integrated framework which supporting virtual prototype}

We need simulating tool to support virtual prototype, and we also need the support of domain design tool and open integrated framework. Integrated framework combines database management ability, network communication ability and process control ability. It not only can realize the uniform management in distributed circumstance, but also virtual prototype.

\subsection{Structure of supporting virtual prototype's integrated framework}

Supporting integrated framework of virtual prototype which based on Client/Server structure and the communication between client and server connects depending on Client/Server of CORBA. Fig.3 shows the structure. 


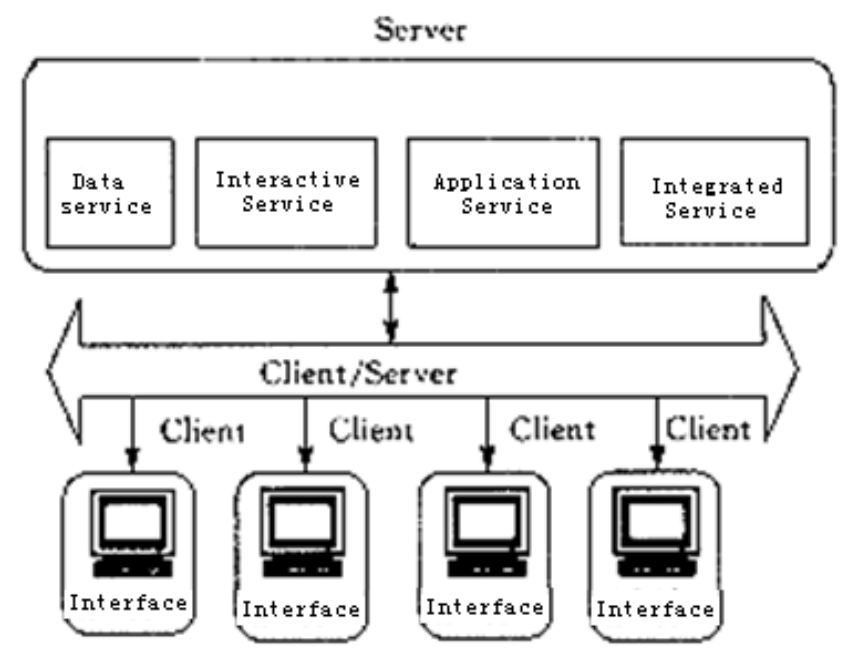

Fig. 3. Integrated framework of virtual prototype

From the point of view of software, it is a hierarchical structure, and the top layer is user server which reflects the main functions supported by virtual prototype system. User can use high-layer service supported by user server by means of client interface, and the bottom-layer structure is not important any more. Every class of service consists of many Agent which can communicate each other flexibility. User service can be divided into four types: Data service, Integrated service, Interchanging service and Application service.

Data service manages and saves the domain data and prototype data, it also generates virtual data. It uses object oriented method to modeling and describes proper model connection components.

Integrated service supports tool integration and team integration. It includes electronics notebook which used to communicate in multi-domain design team.

Interchanging service provides 3D virtual environment and supports product data's vision and interaction.

Application service management provides certain functions for virtual prototype user. It includes some tools which has relationship with virtual prototype design, such as virtual prototype generating tool and virtual prototype simulation tool. It also includes some such certain services as process management, project management, tool management and parallel design plan, and so on.

Above the structures, dada service is the key and difficulty when it used to realize integrated framework of virtual prototype.

\subsection{Data service}

Fig.4 shows the structure which based virtual prototype data service of domain object. We save domain object in Discipline Database (DDB). Virtual prototype is high-layer encapsulation of domain object; it includes multidomain information and provides a digital product simulation model for user. Multi-domain data collection is saved in Prototype Database (PDB) which includes all domain objects, relationship between domain objects, design data and virtual data. User interface receives user's simulation operations by means of simulating interface and converts them into simulating requirements which proposed by virtual prototype. On the other hand, user interface display the simulated result data as figures, so the domain database and prototype database are distributed in many servers. Every Client simulation interface accesses Server where virtual database existed in, and the Server sends service requirement information to the domain object Server. At last, it returns the result to user interface or visual object.[5] 


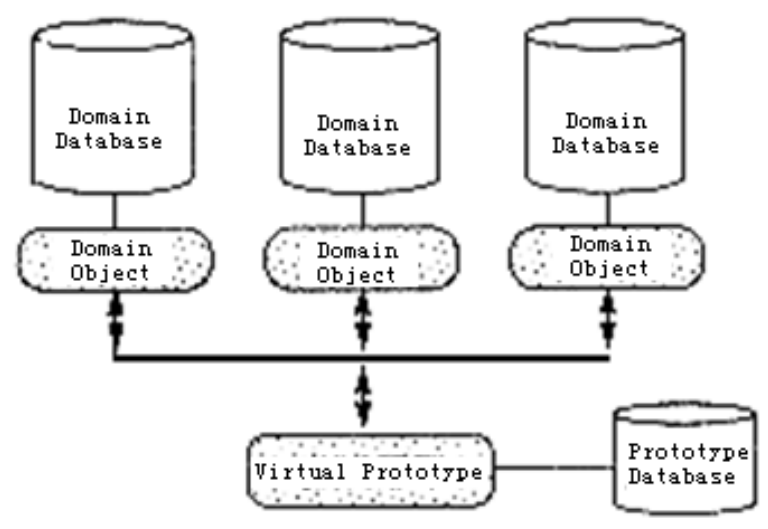

Fig. 4. Virtual Prototype which based on domain object

Domain database provides the following data service functions for virtual prototype: generation and saving of domain object; data service for simulation; data exchanging with some domains; data services for virtual data. Prototype database is generated by object manager, simulation data server and virtual data generator on the data management function. Object manager is mainly used to create, maintain and delete domain objects and vision objects.

\section{Application of virtual prototype technology in low-voltage converter}

This system was developed by CAD/CAM research school Sichuan University, and it has been get a successful application. The construction flow of system is that: First building CAD model of converter; then the built model introduced into 3DS MAX by means of STEP; The virtual model will be saved VRML file by means of VRML97 Exporler pluggable unit, so it can be transmitted in Internet; At last, the processed model will be shown in converter design which based on virtual prototype technology, which providing for users.

\section{Conclusions}

Virtual prototype is a kind of combined technology which consists of multi-subjects and multi-domains. It combines such technologies as $\mathrm{CAD} / \mathrm{CAE}$, parallel engineering, virtual reality, $\mathrm{CSCW}$, artificial intelligence, computer simulation, distributed computer. We are studying the realizing technology of virtual prototype and developing actual virtual prototype environment under the background of electronics equipment design $\&$ application.

\section{Acknowledgment}

We are very grateful to the Jiangsu Province College Natural Science Foundation of China for the support.

\section{References}

[1] Ruan Jingkui, The Research and Development of Integrated CAPP for Panel Dies[J], JOURNAL OF HUBEI AUTOMOTIVE INDUSTRIES INSTITUTE, 2003 17(2)

[2] Niu Wentie, Research on the CAPP system of automobile panel dies[J], JOURNAL OF TIANJIN INSTITUTE OF TECHNOLOGY, 2001 17(4) 
[3] Yao Zhenqiang, ON SYSTEMATIC DESIGN STRATEGY OF MECHANICAL PRODUCTS[J], CHINESE JOURNAL OF MECHANICAL ENGINEERING, 2000 36(6)

[4] Yang Zhong, A New Design and Verification Technique for Virtual Prototypes of UAV Flight Controllers[J], INFORMATION AND CONTROL, 2005 34(1)

[5] Qi Xinmei, Current research situation of mechanical product modeling and its development trend[J], JOURNAL OF HEFEI UNIVERSITY OF TECHNOLOGY, 2000 23(6) 